\title{
A Security Solution Based on IPSec for Smart Substation
}

\author{
Nan Dang ${ }^{1, a}$, Pengjun Zhao ${ }^{2}$ \\ ${ }^{1}$ Electronic Information and Electrical Engineering College,Shang Luo University,ShangLuo \\ 726000, China \\ ${ }^{2}$ Department of Mathematics and Computational Science, Shangluo University, \\ Shangluo 726000 , China \\ adang_lanxin99@163.com
}

Keywords: mart substation, Secure message transmission, Loose coupling strategy, IPSec

\begin{abstract}
A secure solution based on IPSec is proposed for smart substation. It uses the loose coupling strategy in the mode of IPSec to realize the safety transmission of different packet in the substation, according to the different performance requirements and the requirements of the transmission time of smart substation message. The analyses of performance and safety demonstrate the proposed solution satisfies the security requirement of the high performance and can meet the real-time message transmission, improves the transmission efficiency of the network.
\end{abstract}

\section{Introduction}

With the continuous development of power system communication technology, the negative impact of information technology has affected the power system. Substation automation system not only is the basis of the electric power operation, analyze and control link, but also is the most strict safety protection area; Digital substation based on IEC(International Electrotechnical Commission) 61850 standards adopts Ethernet architecture, greatly saves the construction and operation costs, but at the same time, it bright many Security issues. AS the continuous improvement of power system information, information security, confidentiality and reliability problem are increasingly prominent with the importance of SAS increasingly, for example, an attacker could control command opening and closing operation of circuit breaker through forging the remote command at the interior of electric power information network etc[1].Therefore, how to protect the safety of electric power control system and the network information transmission is very important.

According to the transfer time and the performance requirements of a message,IEC62351 、 IEC61850 and IEEE802.1AE set up four different types of message and the corresponding security measures are presented. The four different types of message are SMV message, GOOSE message, GSSE message and MMS message. It will inevitably impact ductility of message transmission and reduce the efficiency of network transmission.

In this paper, we solve the problem based on the loose coupling strategy [2,3] of IPSec to realize the safety of the substation of different packet transmission. The proposed scheme not only meets the security requirement of the high performance but also meets the real-time message transmission, it also improves the transmission efficiency of the network.

\section{Packet classification and the security of the smart substation}

Packet classification of the smart substation. According to the length of packet transmission time,IEC61850 standards splits it into seven categories[4]: (1) A quick message; (2) Tripping message; (3) Medium speed message; (4) Low speed message; (5) The original data packet; (6) File transfer message; (7) Time synchronization message. In order to optimize the decoding process of message, (1) and (2) message map directly to special Ethernet, it is mainly some common object-oriented substation events(GOOSE)/general state of transformer substation events(GSSE); (3),(4), (6) and (7) message are the kind of service-oriented, (5) message is mainly some sample 
measured values (SMV),which is also directly mapped to Ethernet. The IEC62351 standard message format is described in figure 1.

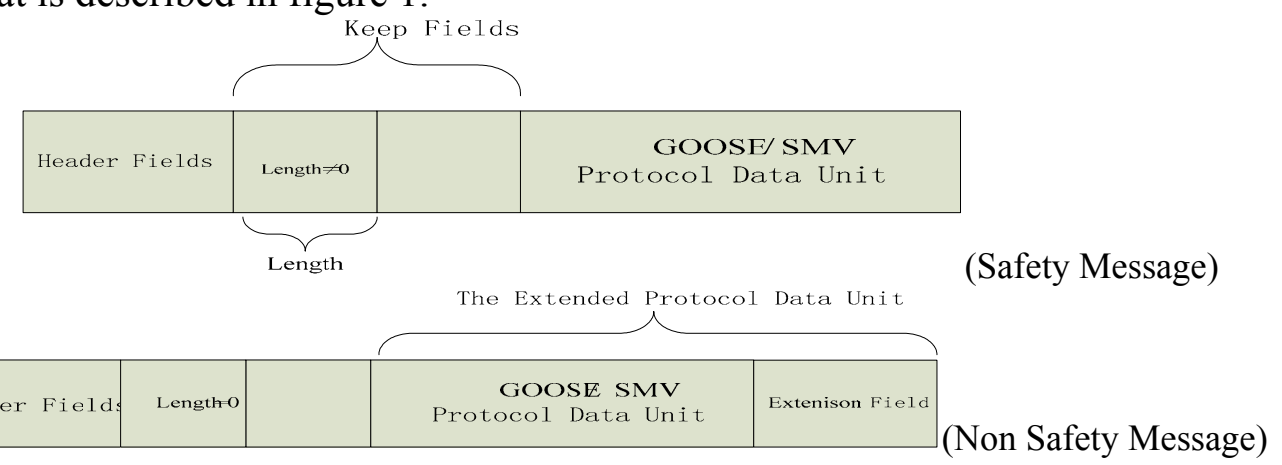

Fig.1 IEC62351 standard message format

IEC62351 standards define the Extension Length through keeping the first field, when the Length $=0$,a message is a security message of ordinary message; If the Length $\neq 0$,it indicates safety message.

Safety measures of smart substation. At substation information security protection, in order to make up for the inadequacy of IEC61850 standard series,WG15 group of the IEC TC57 technical committee establishes IEC62351 standards. The different security measures are listed in table 1.

Table 1 GOOSE/GSSE、MMS、SMV message security measures

\begin{tabular}{cccc}
\hline Category & GOOSE/GSSE & MMS & SMV \\
\hline Encryption Methods & $<4 \mathrm{~ms}$ & Yes & No \\
Identity & No & Yes & No \\
Authentication & certification & $\begin{array}{c}\text { Certification and } \\
\text { encryption }\end{array}$ & Yes \\
\hline
\end{tabular}

Within LAN (Local Area Network),for the real-time and security requirement of GOOSE/GSSE message is higher, so the integrity protection measures is completed through the certification, because the frequent encryption would increase the time delay of the processing of a message, so the encryption method is satisfied with the minimum standards set by the $4 \mathrm{~ms}$ and appropriate encryption algorithm; MMS is connected to the substation's internal and external message, which is vulnerable to malicious attack, so the integrity protection measures is completed through the authentication and encryption of double barrier; SMV message are mainly the measure value of voltage and current sampling by the sensors, it is directly mapped to the Ethernet and obtains the real-time detection status and real-time equipment data, so the data quantity is big, but the security requirements are lower comparing the GOOSE/GSSE and MMS, therefore SMV message can carry out integrity protection rather than encryption.

\section{Basic principle of IPSec}

IPSec provides a standard, robust and inclusive mechanism[5], it is the only one currently which is suitable for all Internet communications security agreement. It defines a set of default and enforce algorithm, which is to ensure that the different implementation of the scheme can be common to each other. IPSec can be nested to provide security services, reduces the threat of the use of IP spoofing for Internet attack, these improv such as e-commerce, extranets and virtual private networks development of applications etc[6].

IPSec protocol includes: Authentication Header(AH)protocol, Encapsulating Security load(ESP)protocol, IKE (Internet Key Exchange) protocol, ISAKMP/Oakley and transcoding. The data source and the connectionless integrity of IP packets sending are provided by the AH,AH can prevent the same IP packet from replaying unceasingly, it doesn't encrypt the packet. In addition to providing the integrity of the data and the data source authentication, ESP encrypts the entire packet and upper level protocol data unit and ensures the security of data.ESP authentication does not include the IP header outside of the ESP header, it includes the transmission mode and tunnel mode 
(see figure 2).The transmission mode is typically used in tunnel implementation of host initialization, it uses the original IP packet header and encrypt the upper head only, so the IPSec head is encapsulated within an IP packet, the IPSec head or is AH header or is the ESP header. The protected IP data head is not be modified. Tunnel model is often used for security gateway, it adds a new IP header outside of the original IP packet header, in the new head, IPSec put the security gateway IP address as the source address, which protect the service machine and a customer's actual address.

\begin{tabular}{|l|c|c|c|c|}
\hline IP Header & IPSec Header & TCP Header & Data \\
\hline IP Header & IPSec Header & IP Header & TCP Header & Data \\
\hline
\end{tabular}

Protected IP packet tunnel mode

Fig.2 ESP transfer mode

\section{Secure solution based on IPSec for smart substation}

The realization of the smart substation combining IPSec message strategy. Smart substation is made of a variety of communication link of transmission message, the message are different in the requirements of the content, length, the complexity of the safety performance and allowing the worst-case transmission time, so it is necessary for different message choose different security solutions. But if IDE on each switches all choose different encryption processing, which can bring not only a lot of time delay but also the difficulty for the IDE interoperability and Key escrow. It is needed to adopt a strategy to meet the different security requirements. For IPSec loose coupling strategy, no safety related nodes of IPSec (e.g, switches, which is connected with IDE)can only forward packets and cannot view the message content. In the Loose Coupling Strategy, all messages sent by a host is not necessarily IPSec packet, it is based on the actual situation of different message transmission. The different security requirements and performance requirements of smart substation messages are shown in table 1 and 2.

(1) the SMV message

SMV message mainly is some samples collected by the sensor measurements, these messages are directly mapped to the Ethernet, real-time detection status and equipment data. SMV packet data obtain transmission requirements are based on the flow; so it does not require for encryption and authentication, it should not be IPSec packet, the integrity is protected by MAC.

(2) GOOSE/GSSE message

GOOSE/GSSE message is directly mapped to a special Ethernet, it is mainly some common object-oriented substation/general substation state messages. GOOSE message is divided into need security protection message and needless security message, need safety message is the fast tripping message in the message 1, so it is used in IPSec traditional mode, which is only need to check the completeness and correctness of the source message. This reduces the time delay and increase the safety of packet data, the specific encryption mode is shown in figure 3.If the GOOSE message is not protected by security, the message handling way consistents with SMV message.

\begin{tabular}{|l|l|l|l|}
\hline IP Header & IPSec Header & TCP Header & GOOSE Packet Data \\
\hline
\end{tabular}

Fig.3 The protected transmission mode GOOSE message data

(3) the MMS message

MMS message is the communication message connected the internal network and external network of substation. It starts from a IDE of dispatching center, transfering from the electric power dispatching network to the substation, then reacheing another IDE by the transformer substation layer and the spacer layer internal, so its safety performance requirements are the highest and it needs real-time requirements. For strengthening the confidentiality of the messages and connectionless integrity, it is necessary for the bi-directional IPSec tunnel model. The concrete encryption mode as shown in figure 4. 


\begin{tabular}{|l|c|c|c|c|}
\hline IP Header & IPSec Header & IP Header & TCP Header & MMS Packet Data \\
\hline
\end{tabular}

Fig.4 Tunnel model protected MMS message data

Performance analysis. The size of the message is 64bit-1518bit in Ethernet, the comparison of transmission delay performance of IPSec protection and no one is shown in table 2.Test environment is selected from a IDE to the end of communication opposite terminal IDE transmitting FTP multiple files, the test method is tested in the FTP multiple files between the host, each file FTP transmission for many times, it is taken the average transmission time finally.

Table 2 With and without IPSec protection substation message transmission delay performance comparison

\begin{tabular}{ccc}
\hline File size (bit) & $\begin{array}{c}\text { No IPSec time } \\
(\mathrm{ms})\end{array}$ & $\begin{array}{c}\text { IPSec time } \\
(\mathrm{ms})\end{array}$ \\
\hline 64 & 0.004956 & 0.0050354 \\
1518 & 1.175514 & 1.194334 \\
10242 & 812 & 825 \\
\hline
\end{tabular}

As can be seen from the test results, when a file is small, the transmission delay is almost unaffected, but when the file is bigger, transmission delay is influencing because of the adding IPSec, the delay of IPSec protection can meet the time requirements of smart substation messages in table 2 .

Safety analysis

The GOOSE/GSSE, MMS and SMV message security measures of IPSec protection is given in table 4.

1) In the IPSec, authentication head AH ensures the integrity and verification services of the IP packet. Based on the data integrity check, it can be judged whether the IP packets is tampered within the process of transmission; Through the verification service and serial number of IPSec, the terminal of system and network equipment can not only filter traffic but also prevent from replaying attack and address spoofing attack. In addition to the data integrity protection and the verification protection, the most important thing is to encrypt data for ESP.

Table 3 The GOOSE/GSSE, MMS and SMV message of IPSec protection

\begin{tabular}{cccc}
\hline category & GOOSE/GSSE & MMS & SMV \\
\hline $\begin{array}{c}\text { Encryption } \\
\text { Methods }\end{array}$ & $\begin{array}{c}\text { IPSec transfer mode } \\
\text { to implement } \\
\text { end-to-end encryption }\end{array}$ & $\begin{array}{c}\text { Two-way IPSec } \\
\text { tunnel } \\
\text { authentication } \\
\text { encryption }\end{array}$ & No \\
$\begin{array}{c}\text { Identity } \\
\text { authentication } \\
\text { Integrity } \\
\text { protection }\end{array}$ & Yes & Yes & No \\
& certification & $\begin{array}{c}\text { Certification and } \\
\text { encryption }\end{array}$ & $\begin{array}{c}\text { through the } \\
\text { MAC }\end{array}$ \\
\hline
\end{tabular}

2)As shown in figure 3,IPSec transfer mode protects the two ends of the data transmission, it is mainly used for the data end-to-end communication(for example, used for the communication between the server and the client), it is routable in the IP. routing path. When using transmission mode, IPSec protects the IP load through the ESP header or the AH header. But transmission mode cannot packet IPSec peer and proceed to NAT (Network Address Translation);For most of the hardware encryption engine, transmission mode of encryption has the lower efficiency than in tunnel mode. For the GOOSE/GSSE message, its real-time transmission and security requirement is higher, so it needs IPSec transfer mode.

3)As shown in figure 4, IPSec tunnel mode encryptes the whole IP packet, when a packet goes through untrusted network, it can protect the communication between different networks. For the MMS message, it connects the internal and external substation, it is very vulnerable to the fake information of denial of service attack. So it needs bidirectional IPSec tunnel mode.

4) IPSec provides a digital certificate service, it can prevent denial attacks. 


\section{Conclusion}

According to the difference of performance and the requirements of the transmission time of GOOSE/GSSE message, MMS message and SMV message, we have proposed a loose coupling strategy of IPSec mode to realize the safety of the substation of different packet transmission in this paper. Performance analysis and security analysis show that the proposed scheme not only meet the security requirement of the high performance but also meet the real-time message transmission and improve the transmission efficiency of the network also.

\section{Acknowledgment}

The authors would like to thank the anonymous referees for their constructive and useful comments that have helped me to improve the presentation of this paper. This work is supported by Research Program Project of Education Department of Shaanxi Provincial Government (Grant No. 2013JK0605) and Scientific Research Foundation of Shangluo University (Grant No. 14SKY-FWDF002).

\section{References}

[1] M.A. Wang. Research on communication security method of digital substation[D]. BEI JING: North China Electric Power University master's thesis, 2011: 1-2.

[2] Perkins C. IP Mobility Support, for Ipv4, RFC3344, 2002, 8.

[3] IETF."IP Encapsulation within IP" , IETF RFC2003, 1996, 5.

[4] IEC 61850-5 Communication networks and systems in substations: Part 5 communication requirements for functions and device models[S].2004.

[5] G.F. Gu, ZHU Guangyu, ZHANG Shiyong,etal. IPSec-based mobile IP security architecture[J]. Computer Science, 2002, 29(12): 83-86.

[6] Y.X. Wang. The technical research and realization of mobile IP traversing Firewal[D].SHENG YANG:hen

Yang University of Technology master's thesis, 2005: 13-14.

[7] PerKins C. IP Mobility Support, RFC2002, 1996, 10. 